\title{
Enhanced Channel Assignment Algorithm for Relaying Nodes in W-CDMA Multihop Cellular Network
}

\author{
Kishana R. Kashwan, Senior Member, IACSIT and S. G. Balakrishnan
}

\begin{abstract}
For newer and emerging communication technologies, it can be quite faster to deploy Multihop cellular networks (MCNs) with increased channel capacity and wider coverage area at comparatively less cost. MCNs greatly reduce the dead-spot and hot-spot problems faced by cellular networks. The channel assignment is a challenge in these networks. Several recent proposals had addressed many channel assignment problems in terms of channel conflicts, node mobility etc. However selecting nodes for relaying during the channel assignment need to be addressed for an effective channel assignment and increased throughput in MCNs. In this paper, a new algorithm, called Enhanced Channel Assignment Algorithm (ECAA) is proposed. The new algorithm enables selection of relaying nodes during channel assignment for a Time Division Duplex (TDD) Wideband Code Division Multiple Access (W-CDMA) MCN. The simulated results show that the proposed scheme ECAA is an improved option channel assignment and resulted in higher throughput.
\end{abstract}

Index Terms-Channel assignment, multihop cellular network, relaying node, throughput.

\section{INTRODUCTION}

With rapid advancement of communication technology, the number of mobile users continues to grow which demands more cellular capacity to fulfill the expected high quality voice communications and high-speed multimedia data transfer services. However, the frequency spectrum is always limited. This has resulted in utilization of radio resources. The use of radio resources increases cellular capacity by dividing a large cell into many smaller cells and then each cell reuses the channel spectrum. In a Code Division Multiple Access (CDMA) cellular system, such as second generation (2G) CDMA or third generation (3G) Wideband Code Division Multiple Access (W-CDMA) system, a small cell size allows a higher interference margin [1] to encounter higher noise level, resulting in a high data rate transmission. This needs that more number of Base Stations (BSs) is to be deployed. Raising more BSs involves higher cost in terms of infrastructure and administration. These networks also suffer

Manuscript received March 17, 2013; revised May 3, 2013.

Kishana R. Kashwan is with the Department of Electronics and Communication Engineering - PG, Sona College of Technology (An Autonomous Institution Affiliated to Anna University), TPT Road, Salem-636005, Tamil Nadu, India, (e-mail: drkrkashwan@ gmail.com and drkrkashwan@sonatech.ac.in).

S. G. Balakrishnan is with the Faculty of Information and Communication Engineering, Anna University, Chennai, India. He is currently with the Department of Computer Science and Engineering, V. M. K. V. Engineering College, Sankari Main Road (NH-47), Periyaseeragapadi, Salem - 636308, Tamil Nadu, India, (e-mail: sgbalakrishnan@ gmail.com) with dead spots having strong shadowing hurdles. To offer coverage in these areas, extra BSs or relay stations may be required. In addition, at some point of time, if the communication traffic demand is less or the number of users is less, then, installing additional BSs or relay stations will remain redundant atleast for some duration at some point of the time. On the other hand, in a $3 \mathrm{G}$ system, various classes of services with a wide range of data rates are provided. Traffic patterns in these networks could be highly dynamic and congested over certain regions such as hot spots. This further discourages to install more BSs since it involves more cost consumption.

Multihop relaying has been demonstrated to be effective in increasing the capacity, coverage, reducing the call blocking probability and decreasing the per node transmission power [2]-[5]. Furthermore, with a conventional system, if the user has a poor channel establishment directly to the base station, they may have no choice but to change location to achieve communication. However, in multihop relaying, mobiles with no good path to any base station may instead relay their calls through its neighbor mobile nodes. Multihop cellular networks (MCNs), which are designed based on the idea of using mobile and fixed terminals to relay signals from source nodes to BSs or destination nodes. Opportunity Driven Multiple Access (ODMA) [6], an ancestor of the current MCN proposal for the 3G Universal Mobile Telecommunications System (UMTS), is designed based on this idea. In ODMA, a long-range transmission is divided into multiple short-range transmissions, which are relayed through intermediate relaying nodes to reduce the interference and, thus, enhance the capacity. Dead-spot and hot-spot problems can also be avoided by relaying signals from dead spots to the BS and from hot spots to neighboring noncongested cells. In addition, no additional BSs are required. A discussion on the economic considerations on the deployment of $\mathrm{MCN}$ can be found in reference [7].

Many researchers have proposed different channel assignment schemes for cellular and ad hoc networks. For cellular networks, the Fixed Channel Assignment (FCA) and Dynamic Channel Assignment (DCA) schemes are normally used [8], [9]. For ad hoc networks also, there are two channel assignment schemes, static and dynamic [10]. A new architecture of integrated cellular and ad hoc relaying (iCAR) system has been proposed [11]. It is based on the logic of diverting the traffic from the more congested cells to the less-congested cells by using the fixed relay stations (RSs) and by relaying the traffic using other frequency bands which may include Industrial, Scientific and Medical (ISM) radio bands.

In yet another research, two new schemes of dynamic 
multihop time-slot allocation of Dynamic Switching Point with Multihop (DSPM) and Maximum Interference First (MIF), for a TDD CDMA system are proposed [12]. DSPM assigns time-slots to mobile nodes in a first-come-first-service basis whereas MIF assigns time-slots based on the energy bit to noise ratio $\left(\mathrm{E}_{\mathrm{b}} / \mathrm{N}_{\mathrm{o}}\right)$. The multihop network architecture consists of six relaying stations located equally apart from each other at a certain distance surrounding the BS. Results show that DSPM increases the data rate for a single user whereas MIF increases the overall system throughput [12]. A new ad hoc cellular (A-Cell) relay architecture has been proposed for 3G W-CDMA [13]. In A-Cell, directional antennae are used to increase spatial reuse. It reduces interference and power consumption. The Global Positioning System (GPS) may be used to facilitate routing protocols [14].

In reference [15], a clustered MCN (cMCN) with a fixed channel assignment is proposed. In $\mathrm{cMCN}$, a macrocell is divided into seven microcells, one center microcell, and six virtual microcells around the center microcell. Each microcell is allocated a fixed set of channels. Each virtual microcell has a wireless dedicated information port (DIP) to assign channel and select relaying path and relay stations for mobile nodes. As the fixed channel assignment cannot cope with temporal changes in the traffic pattern, the authors had proposed the multihop dynamic channel assignment (MDCA) [16]. MDCA assigns channels to mobile nodes based on the interference condition of the channels in a cell with respect to the other interfering cells. The idea is to maximize the channel availability of the system. Results show that MDCA performs better than the fixed channel assignment in terms of system capacity.

There are few more channel assignment schemes available for A-Cell. These include A-Cell Channel Assignment (ACA) [17], Delay-Sensitive Slot Assignment (DSSA) and Random Slot Assignment (RSA) [18]. The ACA is formulated as a linear optimization problem to optimize the channel reuse in A-Cell. However, it does not address the packet delay issue. DSSA is a heuristic channel assignment scheme designed based on ACA to take into account packet delay. However, it applies only to an Omnidirectional antennae environment, and not suitable for a directional antennas environment. RSA is similar to DSSA except that channels or timeslots are assigned randomly. Obviously, RSA cannot guarantee minimum delay. In fact, simulation results show that RSA has a lower performance than that of DSSA in terms of throughput and packet relaying delay [18].

In reference [19], The Mobility and Load aware Routing scheme (MLR) aims to find a stable route with a long lifetime by letting the intermediate nodes to decide whether to broadcast or drop the RREQ packets based on its speed and routing load. To make such decision, MLR uses the Markovian Decision Process (MDP) tool trying to find the best action that the node can take to maximize the overall network performance. MLR modifies the route discovery phase specifically the propagation of RREQ packets of the original reactive protocols. Route replay and maintenance is the same as the original protocol.

In references [20] and [21], optimal channel assignment (OCA) scheme for ad hoc TDD W-CDMA MCN and a heuristic channel assignment scheme called minimum slot waiting first (MSWF) is discussed. A time division duplex (TDD) wideband code division multiple accesses (W-CDMA) MCN are proposed to minimize packet delays. The task of finding a channel assignment is to ensure that no signal collision, channel conflict or time-slot conflict occur and also, the total packet relaying delay is minimized. This approach is quite effective for W-CDMA schemes.

In reference [22], Mobility Aware Channel Assignment (MACA) is proposed. The first task is to get input or in other words gather all information of the current setup path and neighboring existing setup path to the current node call request. Second task is to find transmission zones of all mobile nodes of currently setup path and also its neighboring existing setup path. Importantly the third task is to eliminate the channels, which could possibly create channel conflicts due to random node mobility within current path setup and also it's neighboring existing path setup. The fourth main task of MACA algorithm is to choose channels from remained ones after eliminating conflicting channels due to mobility. Here, the channel selection simultaneously require relaying delay criterion. The least relaying delay in the current set up path is selected.

In the recent years many channel assignment schemes has been proposed for increasing capacity, coverage, decreasing node transmission power and reducing packet delay in the MCNs. However, the importance of selecting relaying nodes in MCNs environment is not well addressed. In this paper, relaying node selection during the channel assignment for MCNs to offer best channel assignment was considered.

In this paper, the authors have proposed an Enhanced Channel Assignment algorithm (ECAA) for MCNs with objective of offering best channel assignment with increased throughputs in a time division duplex (TDD) W-CDMA MCN. The main focus was on the selection of relaying nodes during channel assignment in TDD WCDMA MCNs, where nodes (NDs) are equipped with directional antennas. However this scheme could also be used for nodes equipped with omnidirectional antennas. These networks allow quick deployment at low cost. Though MCNs have many advantages, they also pose many challenges. The most important of which is improper relaying nodes' selection during channel assignment. This leads to decreased throughput and inefficient channel assignment. Therefore, these need a better and more intelligent channel assignment scheme.

The paper is organized as follows: The Section II examines the main issue of multihop cellular networks channel assignment for TDD W-CDMA MCN. In Section III, the ECAA scheme is presented. The Section IV discusses implementation parameters of ECAA and the results are presented in Section V.

\section{RELAYING NODES PROBLEM IN MCNS}

The channel assignment is represented by a pair of variables, time-slot $T$ and code $C$. The channel conflict may occur while assigning a channel to establish a connection with a mobile node. However relaying nodes in MCNs is playing an important role for carrying the voice or data traffic between 
mobile nodes. The relaying node selection issue is also main focus of this research work. A relaying node may have several relaying points to offer multiple data transfer connections. Highly overloaded relaying node may cause of data loss due to buffer overflow, data transfer delay due to heavy traffic flow through a particular relaying node and connection loss because of relaying node mobility. In MCNs, a source node may have several paths and also have equal number of hop counts in a few of the paths to establish connection to base station for its uplink transmission. This is indicated by dashed line in Fig.1. However, the shortest path must to given for a given channel assignment task. In this node M5 have two equivalent paths to reach its base station are $\mathrm{M} 5 \rightarrow \mathrm{M} 4 \rightarrow \mathrm{M} 3 \rightarrow \mathrm{BS}$ and $\mathrm{M} 5 \rightarrow \mathrm{M} 4 \rightarrow \mathrm{M} 2 \rightarrow \mathrm{BS}$. Here node M3 has two connections through its relaying points and node M2 has a single connection through its relaying point. During channel assignment, if the first path is selected then node M3 may increased its relaying points resulting in poor path selection for channel assignment, if node 2 is chosen and supposing that the next relaying node may have high relaying points, then again it leads to a poor path selection for channel assignment. However, choosing a single path with availability of multiple equivalent paths to the channel assignment algorithm may not considerably increase the cell throughput. It also may leads to ineffective channel assignment.

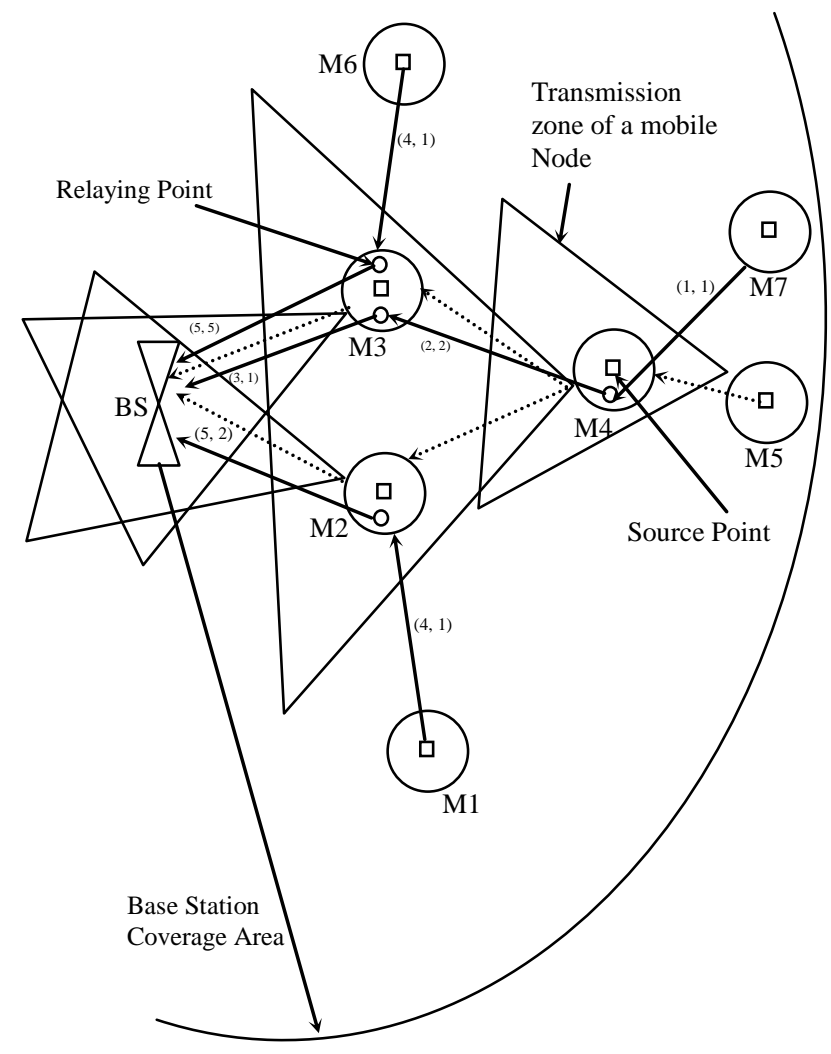

Fig. 1. MCNs Topology with relaying nodes

\section{ECAA SCHEME}

In this section, the authors propose a scheme called Enhanced Channel Assignment algorithm (ECAA). The scheme resides in radio network controller (RNC), which, in turns, controls many base stations (BS). The RNC keeps complete information of a cell, which includes routing mechanism, location, transmission zones of mobile node, data rate and channel assignment of all mobile nodes involved in communications. The ECAA scheme is designed based on two principles, which are summarized as follows.

- Compute the relaying points for a proposed source node in the set of equivalent available paths and choose the best path for channel assignment purpose.

- Find the channels which avoid channel conflicts and also give less relaying delay in the current setup path.

The proposed scheme, ECAA can be explained in two different stages of process.

TABLE I: ALGORITHM STEPS FOR STAGE-I OF ECAA Simplified simulation flow of ECAA algorithm

1. Input multiple equivalent paths ( 1 to $N$ ) where $N$ is the last path

2. Input a path $I$ (where $I=1)$

3. The last hop node to the BS := current node (NDC)

4. Count the number of relaying points (RP) in the NDC and increase RP index by 1

5. Reserve a channel $(\mathrm{CH})$ to NDC, Propose a not-tried available $\mathrm{CH}(\mathrm{T}, \mathrm{C})$, with index of $\mathrm{T}$ is largest in value If NDC not receiving on $\mathrm{T}$, and If $\mathrm{NDs}$ in $\mathrm{TZ}$ of NDC not receiving on $\mathrm{CH}$, then $\mathrm{CH}$ is reserved for NDC, else $\mathrm{CH}$ is removed and $\mathrm{CH}$ reservation fails, else All $\mathrm{CHs}$ in $\mathrm{T}$ are removed and $\mathrm{CH}$ reservation fails endif

6. NDC:= node next hop (NDN) and successor of NDC:= NDC

7. Count the number of relaying points (RP) in the NDC and add the value in RP index

8. Reserve a channel $(\mathrm{CH})$ to NDN

Propose a not-tried available $\mathrm{CH}$ with $\mathrm{T}$ nearby to $\mathrm{CH}$ of NDN If NDC not receiving on T and NDN not transmitting on or assigned with $\mathrm{T}$, then

If NDs is on other routes with TZ and NDN falls, not transmitting on $\mathrm{CH}$, then

TZ in which NDN falls, not transmitting on this $\mathrm{CH}$ and NDs in $\mathrm{TZ}$ of NDC not receiving on $\mathrm{CH}$, then

$\mathrm{CH}$ is reserved for NDC, else

$\mathrm{CH}$ is removed and $\mathrm{CH}$ reservation fails, endif, else

All $\mathrm{CHs}$ in $\mathrm{T}$ are removed and $\mathrm{CH}$ reservation fails Endif

While $\mathrm{CH}$ reservation fails and not-tried available $\mathrm{CHs}$ exists If $\mathrm{CH}$ reservation fails, return

Repeat steps 6, 7 and 8 until the source node is reached If $I<=N$, then

Increment $I$ by 1 and continue step 3, else

Update reserved channel information and RP values in BS table and nodes table

9. Select the best path from source node path index

10. Exit the algorithm 


\section{A. Stage - I}

During stage $I$, the number of relaying points for each path of the source node path index and channel reservation for each path in the source node path index of the proposed node is determined by computation according to the steps sequence given in the algorithm as listed in Table I.

\section{B. Stage - II}

During the second stage, a best path is chosen for input that have been chosen based on relaying points and further, the reserved channels for that path are assigned to the proposed node connection. The sequence steps for stage II are listed in Table II as shown below.

TABLE II: ALGORITHM STEPS FOR STAGE-II OF ECAA

Simplified simulation flow of ECAA algorithm

1. Input a best path that have been chosen based on relaying points computed from stage-I process.

2. Assigned Reserved channels to the selected path of the proposed node

3. Update assigned channels information in BS table and nodes table

4. Exit the algorithm

\section{Execution Steps of ECAA}

Algorithm steps as listed in Table I for stage-I demonstrate 10 major steps. During first step, inputs are received for the multiple equivalent paths available for source node. The iteration of all paths for computing number of relaying points in each path is performed during the algorithms' second step. Channels are reserved starting from the last-hop node of the path in reverse sequence till the source node is approached. A last-hop node is the node nearby to the BS on a path and the same is selected in step 3. The last-hop node becomes the current node in next operation. For example, in Fig. 1, BS->M3->M4>M5 is a path where node M3 is the last-hop node which is also the current node and is located nearest to the base station.

During execution of step 4, counting of the number of relaying points (RP) in the NDC are done and also RP index is updated by incrementing it by 1 .

A channel is proposed to the current node during step 5, which is a last-hop node. An available channel (a time-slot T and code $C$ pair) in which the time-slot has the largest index is proposed to the current node. If the current node is receiving on the proposed time slot then the channel is not reserved and all the channels in time-slot $\mathrm{T}$ are removed. Further if nodes in the transmission zone of the current node are receiving on the proposed channel then the channel is removed else the proposed channel is reserved for the current node. This may in the case when the channel reservation fails in search of a not-tried available channel in which the time-slot has the largest index for the current node. If the channel satisfies the conditions listed in step 5, the channel is temporarily reserved to the current node and the channel reservation is successful. The channel reservation process continues with Step 6, otherwise, the channel reservation is a failure and the channel reservation process is terminated.

In Step 6, the current node becomes the next-hop node whereas the descendant of current node assumes the position of the current node. This can be illustrated with the help of Fig. 1, as shown there, the mobile node M4 becomes the current node while mobile node M3 becomes the next-hop node in the process of continuing the execution of steps in ECAA algorithm.

The execution of step 7 counts the number of relaying points (RP) in the NDC and increments the value in RP index to update the same for the next operation.

A channel is reserved to the current node during step 8. An available channel, having a time-slot (index) which is consecutive and is nearby to the time-slot (index) of the channel reserved to the next-hop node, is proposed to be the current node. The proposed channel is then tested by using four conditions specified in step 8. If no conditions are violated, the channel is temporarily reserved to the current node. If first and second conditions are violated, all the channels in a time-slot of the proposed channel are removed. If only the third and fourth conditions are violated, the proposed channel is eliminated. However in both cases, the channel reservation fails and the algorithm continues to search a not-tried available channel for the current node. If all the not-tried available channels do not satisfy the conditions and the channel reservation still fails, the channel reservation process is finished. If channel reservation is a success, steps 6 , 7 and 8 are repeated for channel reservation for the next current node. This iterative process continues until the source node is reserved for a channel or a channel assignment fails. In step 9, the best path is selected from source node path index by the process of computation based on relaying points. The ECAA algorithm finally exits at step 10 .

In Step 6, if channel assignment is a success, i.e., each node on the path is temporarily assigned a channel, the call (connection) is accepted and the channel information table of the nodes and the BS are updated, otherwise, the temporary assignments are removed.

There are four major steps for ECAA for stage-II. During step1, the input is presented with a best path that has been chosen based on relaying points computed at stage-I process. At step 2, a reserved channel is assigned to the selected path of the proposed node. The step 3 updates assigned channel information in BS table and nodes tables. Finally, at step 4, the algorithm exits execution sequence.

The node neatest to the BS normally encounters increased channel conflicts due to many channels communicating simultaneously. This can be avoided by choosing further node as the last-hop node. As a relaying node on the path is far away from the BS, the fewer number of channels at the relaying node will communicate with it. Thus, many channels are available for assignment purpose. This helps in increasing the probability suitable channel assignment.

\section{Simulation TeStS AND RESUlts}

The test packet simulation for proposed algorithm has been carried out by selecting four mobile nodes M4, M5, M6 and 
M7 as shown in Fig. 1 with single BS. The topology is shown by Fig. 1 was considered for the simulation tests for static nodes configuration. As shown in Fig. 1, M6 node has been assigned the channels $(4,1)$ for establishing a connection to BS through relaying node M3. The M6 is assigned $(4,1)$ and $(5,5)$ channels for the connection to BS through relaying node M3. Nodes M4 and M6 transmit to BS through relaying node M3 simultaneously. Simulated results show that packet reception at base station is good for both the connections.

The simulation parameters chosen for experimental tests are summarized in the TABLE III. The parameters are selected for two kinds of hops, single and multi-hop. The throughput analysis simulation is illustrated by graphical visuals as shown in Fig. 2. Packet delivery ratio and throughput of the cell increase for simulated results of ECAA scheme whereas for single-hop, the simulation tests showed that the packet delivery ratio and throughput was poor. The throughput for ECAA is about 6 times compared to that of single hop case.

TABLE III: THE SiMUlator PARAMETER CHOSEN

\begin{tabular}{lll}
\hline \hline Parameters & Single-hop & Multi-hop \\
\hline No. of codes / & 1 & 5 \\
Time - slot & & \\
No. of Time - slots / frame & 5 & 5 \\
No. of Time - slots for uplink & 5 & 5 \\
Mobile transmission range & 1100 meter & 250 meter \\
Mobile capacity & $207 \mathrm{kbps}$ & $1035 \mathrm{kbps}$ \\
Hop count & 1 & 4 \\
Data rate/code & $13.8 \mathrm{kbbs}$ & $13.8 \mathrm{kbps}$ \\
No. of source nodes & 50 & 50 \\
Antenna (directional) & Beam angle 45 & Beam angle 45 \\
& degree & degree \\
Connection request (call) & 0.5 connections & 0.5 connections \\
& /min. & /min. \\
Connection request ( data & 0.5 connections / & 0.5 connections \\
transfer) & min. & / min. \\
Connection (call / data & 1 minute & 1 minute \\
transfer) holding time & & 10 minutes \\
Duration & 10 minutes & \\
\hline \hline
\end{tabular}

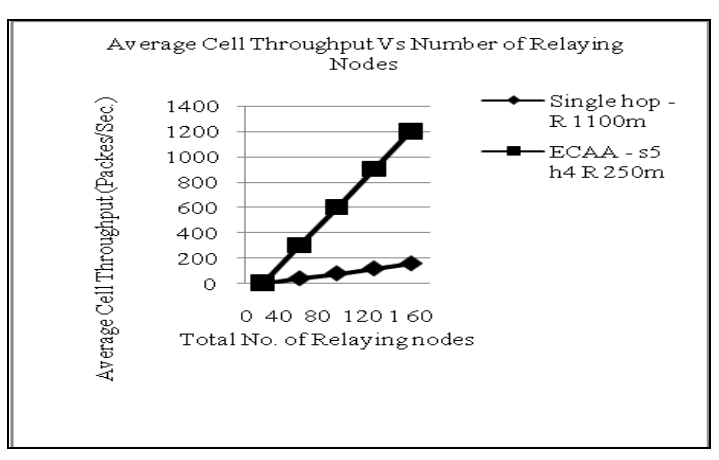

Fig. 2. Throughput analysis of cell

\section{CONCLUSION}

A new scheme called ECAA is proposed in this for TDD WCDMA MCNs for taking into consideration of mobile nodes relaying points while assigning channel. It appears to be suitable for dead spot and hot spot problems in TDD WCDMA MCN. The ECAA algorithm had offered a better quality of service in the MCNs due to improved channel assignments and reduced channel conflicts. Simulations tests were carried out to validate the theoretical proposing of algorithm. It had resulted in a higher throughput; approximately 6 times as compared to single hop networks. The future work may be focused towards further validating the algorithm for more advanced simulation tests and real time wireless communication systems.

\section{REFERENCES}

[1] H. Holma and A. Toskala, WCDMA for UMTS, Radio Access for Third Generation Mobile Communications, third ed., John Wiley \& Sons, 2004.

[2] Y. D. Lin and Y. C. Hsu, "Multi-hop cellular: A new architecture for wireless communications," in Proc. 19th IEEE annual conference on computer and communications, INFOCOM, March 2000, vol. 3, pp. 1273-1282.

[3] A. Radwan and H. S. Hassanein, "Capacity enhancement in CDMA cellular networks using multi-hop communication," in Proc. 11th IEEE symposium on computers and communications, June, pp. 832837.

[4] K. J. Kumar, B. S. Manoj, and C. Siva Ram Murthy, "RT-MuPAC: A new multi-power architecture for voice cellular networks," Computer Networks, vol. 47, pp. 105-128, 2005.

[5] H. Wu, C. Qiao, S. De, and O. Tonguz, "Integrated cellular and ad hoc relaying systems: iCAR," IEEE Journal on Selected Areas in Communications, vol. 19, pp. 2105-2115, 2001.

[6] Third Generation Partnership Project (3GPP), Technical Specification Group Radio Access Network, Opportunity Driven Multiple Access (ODMA), Sophia Antipolis, Valbbonne (3G TR 25.924 Version 1.0.0), 1999.

[7] X. J. Li, B.-C. Seet, and P. H. J. Chong, "Multihop Cellular Networks: Technology and Economics," Computer Networks, vol. 52, no. 9, pp. 1825-1837, June 2008.

[8] I. Katzela and M. Naghshineh, "Channel assignment schemes for cellular mobile telecommunication systems: A comprehensive survey," IEEE Personal Communications, vol. 3, no. 3, pp. 10-31, 1996, doi:10.1109/98.511762.

[9] P. H. J. Chong and C. Leung, "A network-based dynamic channel assignment scheme for TDMA cellular systems," International Journal of Wireless Information Networks, vol. 8, no. 3, pp. 155-165, doi:10.1023/A:1012378931278.

[10] D. Grace, T. C. Tozer, and A. G. Burr, "Reducing call dropping in distributed dynamic channel assignment algorithms by incorporating power control in wireless ad hoc networks," IEEE Journal on Selected Areas in Communications, vol. 18, no. 11, pp. 2417-2428, 2000, doi:10.1109/49.895046.

[11] H. Wu, C. Qiao, S. De, and O. Tonguz, "Integrated cellular and ad hoc relaying systems: iCAR," IEEE Journal on Selected Areas in Communications, vol. 19, no. 10, pp. 2105-2115, 2001, doi:10.1109/49. 957326.

[12] M. Yang and P. H. J. Chong, "Time Slot Allocation Schemes for Multihop TDD-CDMA Cellular System," in Proc. IEEE Wireless Comm. and Networking Conf. (WCNC '07), March, 2007, pp. 3099-3104.

[13] A. M. Safwat, "A-Cell: A Novel Multi-Hop Architecture for 4G and 4G+ Wireless Networks," in Proc. IEEE Vehicular Technology Conf. (VTC '03), Oct. 2003, pp. 2931-2935.

[14] C. K. Toh, Ad Hoc Mobile Wireless Network, Protocols and Systems, first ed. Prentice-Hall, 2001.

[15] X. J. Li and P. H. J. Chong, "Performance Analysis of Multihop Cellular Network with Fixed Channel Assignment," Wireless Networks, vol. 16, pp. 511-526, Feb. 2010.

[16] X. J. Li and P. H. J. Chong, "A Dynamic Channel Assignment Scheme for TDMA-Based Multihop Cellular Networks," IEEE Trans. Wireless Comm., vol. 7, no. 6, pp. 1999-2003, June 2008.

[17] A. M. Safwat, "ACA: Channel Assignment in Ad Hoc, 4G and Beyond Wireless Networks with Directional Antennas," in Proc. IEEE Int'l Conf. Comm. (ICC '04), June 2004, pp. 3143-3147.

[18] M. Al-Riyami, A. M. Safwat, and H. S. Hassanein, "Channel Assignment in MultiHop TDD W-CDMA Cellular Networks," in Proc. IEEE Int'l Conf. Comm. (ICC '05), May 2005, pp. 1428-1432. 
[19] Y. Khamayseh, G. Obiedat, and M. B. Yassin, "Mobility and Load aware Routing protocol for ad hoc networks," Journal of King Saud University - Computer and Information Sciences, 6 May 2011, pp. 106-113.

[20] Y. H. Tam, R. Benkoczi, H. S. Hassanein, and S. G. Akl, "Optimal Channel Assignment in Multi-Hop Cellular Networks," in Proc. IEEE GLOBECOM, pp. 731-735, Nov. 2007.

[21] Y. H. Tam, R. Benkoczi, H. S. Hassanein, and S. G. Akl, "Channel Assignment in Multi-hop Cellular Networks: Minimum Delay," IEEE Transactions on Mobile Computing, vol. 9, issue 7, pp. 1022-1034, July 2010.

[22] K. R. Kashwan and S. G. Balakrishnan, "Channel Assignment Algorithm for Mobile Nodes in Multihop Cellular Network," in Proc. ICECT 2012, pp. 200-204, April 2012.

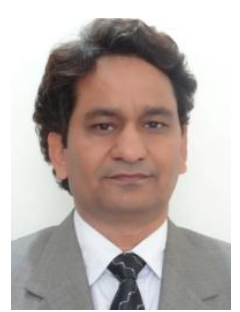

Kishana R. Kashwan has received the degrees of M.Tech. in Electronics Design and Technology and Ph.D. in Electronics and Communication Engineering from Tezpur University (a central university of India), Tezpur, India, in 2002 and 2007 respectively. Presently he is a Professor and DEAN of Post Graduate Studies in the department of Electronics and Communication Engineering (Post Graduate Studies), Sona College of Technology (An Autonomous Institution Affiliated to Anna University), TPT Road, Salem - 636005, Tamil Nadu, India. He has published extensively at international and national level and has travelled to many countries. His research areas are VLSI Design, Communication Systems, Circuits and Systems and $\mathrm{SoC} / \mathrm{PSoC}$. He is also director of the Centre of Excellence in VLSI Design and Embedded SoC at Sona College of Technology. He is a member of Academic Council, Research Committee and Board of Studies of Electronics and Communication Engineering at Sona College of Technology. He has successfully guided many scholars for their master's and doctoral theses. Prof. Kashwan has completed many funded research projects. Currently, he is working on a few funded projects from Government of India. Dr. Kashwan is a member of IEEE, IASTED and Senior Member of IACSIT. He is life member of ISTE and Member of IE (India).

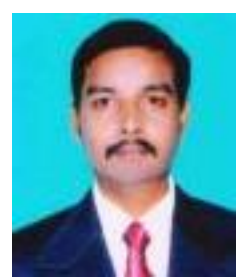

Balakrishnan S. G. is an assistant professor at the V.M.K.V. Engineering College, India. His research interests are in the design of algorithms for multihop cellular networks. He is pursuing Ph.D in Anna University, India. $\mathrm{He}$ has a Master degree in Information Technology from Vinayaka Missions University, India and Bachelor degree in Computer Science and Engineering from Periyar University, India. He is a member of the CSI. He is currently involved in research work of wireless sensor networks and its' applications for real-time communication systems. His main research interests are focused on computer science and engineering, information and communication technology. 